\title{
ON A THEOREM OF G. W. MACKEY
}

\author{
BY ROBERT J. BLATTNER ${ }^{1}$
}

\section{Communicated by A. M. Gleason, June 6, 1962}

Let $G$ be a locally compact group, $H$ a closed subgroup of $G$, and $L$ a unitary representation of $H$ on the Hilbert space $v$. Let $U^{L}$ be the representation of $G$ induced by $L$ (see [1] for the definitions and notations of the present note). For $h \in C_{0}(G / H)$ and $f \in \mathcal{H C}\left(U^{L}\right)$, set $\left(E^{L}(h) f\right)(x)=h(\pi(x)) f(x)$, where $\pi$ is the natural projection of $G$ on $G / H$. Then $E^{L}$ is a $*$-homomorphism of the commutative *-algebra $C_{0}(G / H)$ (under the pointwise operations) into $\mathscr{L}\left(\mathfrak{H C}\left(U^{L}\right), \mathfrak{H C}\left(U^{L}\right)\right.$ ). Let $M$ be another unitary representation of $H$ on a Hilbert space $w$ and form $U^{M}$ and $E^{M}$. If $T \in R(L, M)$, the space of operators intertwining $L$ and $M$, define $T^{\sim}$ by setting $(T \sim f)(x)=T f(x), f \in \mathcal{K}\left(U^{L}\right)$.

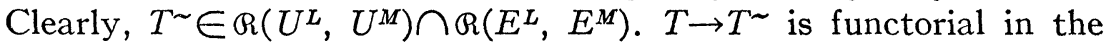
sense that it commutes with operator multiplication and adjunction and sends any identity (resp. zero) operator onto an identity (resp. zero) operator.

The following theorem is due to G. W. Mackey in the case that $G, \mathcal{V}$, and $\mathscr{W}$ are separable [2, Theorem 6.4]. His techniques are measure theoretic and do not seem suited to the nonseparable case.

Theorem. $T \rightarrow T^{\sim}$ is an isomorphism onto.

Proof. (a) Linearity is clear. Suppose $T_{1}^{\sim}=T_{2}^{\sim}$. For $\phi \in C_{0}(G)$, $v \in \mathcal{V}$, form

$$
\epsilon_{L}(\phi, v)(x)=\int_{H} \phi(\xi x) \delta(\xi)^{-1 / 2} \Delta(\xi)^{1 / 2} L \bar{\xi}^{-1} v d \xi,
$$

as in [1]. (All integrations in this paper are with respect to right Haar measure. $\Delta$ (resp. $\delta$ ) is the modular function of $G$ (resp. $H)$.) $\epsilon_{L}(\phi, v)$ $\in \mathscr{H C}\left(U^{L}\right) . \epsilon_{M}(\phi, w), w \in \mathscr{W}$ defined similarly, $\in \mathscr{H}\left(U^{M}\right)$. Moreover, $T_{i}^{\sim} \epsilon_{L}(\phi, v)=\epsilon_{M}\left(\phi, T_{i} v\right)$. Therefore $\epsilon_{M}\left(\phi, T_{1} v\right)(e)=\epsilon_{M}\left(\phi, T_{2} v\right)(e)$ for all $\phi \in C_{0}(G)$. Letting $\phi \mid H$ approach the $\delta$-measure, we obtain $T_{1} v=T_{2} v$, $v \in v$. Therefore $T \rightarrow T^{\sim}$ is one-to-one.

(b) Suppose $L=M$. To show the mapping is onto, it is sufficient to show that if $T_{0} \in R\left(U^{L}, U^{L}\right) \cap R\left(E^{L}, E^{L}\right), 0 \leqq T_{0} \leqq I$, then $T_{0}=T^{\sim}$ for some $T \in R(L, L)$.

We define a linear map $S$ of $C_{0}(G) \otimes \mathcal{H C}\left(U^{L}\right)$ into $V$ by setting $S(\phi \otimes f)=\int_{G} \phi(y) f\left(y^{-1}\right) d y$. Now $S(\phi \otimes \epsilon(\psi, v))=\epsilon\left(\psi^{*} \phi, v\right)(e)$. Therefore

${ }^{1}$ National Science Foundation Fellow. 
$S(\phi \otimes \epsilon(\psi, v)) \rightarrow v$ as $\psi * \phi \mid H \rightarrow$ the $\delta$-measure, and the range of $S$ is dense in $v$. Again,

$$
\begin{aligned}
(S(\phi & \otimes f), S(\psi \otimes g)) \\
& =\iint \phi(y)[\psi(x)]^{-}\left(f\left(y^{-1}\right), g\left(x^{-1}\right)\right) d x d y \\
& =\iint \phi\left(y x^{-1}\right)\left[\psi\left(x^{-1}\right)\right]^{-} \Delta(x)^{-1}\left(\left(U_{y^{-1}}^{L}\right)(x), g(x)\right) d x d y \\
& =\int\left(E^{L}(\{\phi, \psi\}(\cdot, y)) U_{y^{-1}}^{L}, g\right) d y,
\end{aligned}
$$

where $\{\phi, \psi\}$ is the function in $C_{0}(G / H \times G)$ defined by

$$
\{\phi, \psi\}(\pi(x), y)=\int_{H} \phi\left(y(\xi x)^{-1}\right)\left[\psi\left((\xi x)^{-1}\right)\right]^{-} \Delta(\xi x)^{-1} d \xi .
$$

This follows from the fact that if $f, g \in \mathfrak{F}\left(U^{L}\right)$ and if $h \in C_{0}(G)$, then $\left(E^{L}(\tau h) f, g\right)=\int h(x)(f(x), g(x)) d x$, where $(\tau h)(\pi(x))=\int_{H} f(\xi x) d \xi$. For any $\lambda \in C_{0}(G / H \times G)$, we define $\Phi(\lambda) \in \mathscr{L}\left(\mathfrak{H}\left(U^{L}\right), \mathfrak{K}\left(U^{L}\right)\right)$ by setting

$$
\Phi(\lambda)=\int E^{L}(\lambda(\cdot, y)) U_{y^{-1}}^{L} d y .
$$

We have shown $(S(\phi \otimes f), S(\psi \otimes g))=(\Phi(\{\phi, \psi\}) f, g)$.

Now

$$
\begin{aligned}
\left\|S\left(\sum \phi_{i} \otimes T_{0}^{1 / 2} f_{i}\right)\right\|^{2} & =\sum_{i j}\left(\Phi\left(\left\{\phi_{i}, \phi_{j}\right\}\right) T_{0}^{1 / 2} f_{i}, T_{0}^{1 / 2} f_{j}\right) \\
& =\sum_{i j}\left(T_{0} \Phi\left(\left\{\phi_{i}, \phi_{j}\right\}\right) f_{i}, f_{j}\right),
\end{aligned}
$$

because $\Phi(\lambda)$ commutes with $T_{0}^{1 / 2}, \lambda \in C_{0}(G / H \times G)$. Adding this equation to the equation got from it by replacing $T_{0}$ with $I-T_{0}$, we obtain

$$
\begin{aligned}
\left\|S\left(\sum \phi_{i} \otimes T_{0}^{1 / 2} f_{i}\right)\right\|^{2} & \leqq\left\|S\left(\sum \phi_{i} \otimes T_{0}^{1 / 2} f_{i}\right)\right\|^{2}+\left\|S\left(\sum \phi_{i} \otimes\left(I-T_{0}\right)^{1 / 2} f_{i}\right)\right\|^{2} \\
& =\sum_{i j}\left(\Phi\left(\left\{\phi_{i}, \phi_{j}\right\}\right) f_{i}, f_{j}\right)=\left\|S \sum \phi_{i}\left(\otimes f_{i}\right)\right\|^{2} .
\end{aligned}
$$

Since $\sum \phi_{i} \otimes f_{i} \rightarrow \sum \phi_{i} \otimes T_{0}^{1 / 2} f_{i}$ is linear, there is a unique bounded linear operator $T_{1}$ on $\vartheta$ such that $T_{1} S(\phi \otimes f)=S\left(\phi \otimes T_{0}^{1 / 2} f\right)$, all $\phi$ and f. But $L_{\xi} S(\phi \otimes f)=\delta(\xi)^{-1 / 2} \Delta(\xi)^{1 / 2} S\left(\left(R_{\xi} \phi\right) \otimes f\right)$, all $\xi \in H$, where $\left(R_{\xi} \phi\right)(x)=\phi(x \xi)$. It follows that $T_{1} \in R(L, L)$.

Let $T=T_{1}^{*} T_{1} \in R(L, L)$. We have 
$(S(\phi \otimes T \sim f), S(\psi \otimes g))$

$$
\begin{aligned}
& =(T S(\phi \otimes f), S(\psi \otimes g))=\left(S\left(\phi \otimes T_{0}^{1 / 2} f\right), S\left(\psi \otimes T_{0}^{1 / 2} g\right)\right) \\
& =\left(\Phi(\{\phi, \psi\}) T_{0}^{1 / 2} f, T_{0}^{1 / 2} g\right)=\left(\Phi(\{\phi, \psi\}) T_{0} f, g\right) \\
& =\left(S\left(\phi \otimes T_{0} f\right), S(\psi \otimes g)\right) .
\end{aligned}
$$

Hence $\int \phi(y)(T \sim f)\left(y^{-1}\right) d y=\int \phi(y)\left(T_{0} f\right)\left(y^{-1}\right) d y$ for all $\phi \in C_{0}(G)$. It follows that $T \sim f=T_{0} f$, all $f$, and $T_{0}=T^{\sim}$.

(c) General case. Let $T_{0} \in \Re\left(U^{L}, U^{M}\right) \cap \Re\left(E^{L}, E^{M}\right)$. Now $U^{L \oplus M}$ $\cong U^{L} \oplus U^{M}$ in a canonical way. Using this isomorphism and matrix notation, set

$$
T_{1}=\left(\begin{array}{ll}
0 & 0 \\
T_{0} & 0
\end{array}\right)
$$

$T_{1} \in R\left(U^{L \oplus M}, U^{L \oplus M}\right) \cap \Omega\left(E^{L \oplus M}, E^{L \oplus M}\right)$. Thus $T_{1}=T_{2}^{\sim}$ where $T_{2}$ $\in \Re(L \oplus M, L \oplus M)$. Write

$$
T_{2}=\left(\begin{array}{ll}
* & * \\
T & *
\end{array}\right) .
$$

$T \in R(L, M)$. It is easily seen that $T_{0}=T^{\sim}$.

REMARK. It follows immediately from this theorem that $\left(U^{L}, E^{L}\right)$ is equivalent to $\left(U^{M}, E^{M}\right)$ if and only if $L \cong M$ and that $\left(U^{L}, E^{L}\right)$ is irreducible if and only if $L$ is irreducible.

\section{REFERENCES}

1. R. J. Blattner, On induced representations, Amer. J. Math. 83 (1961), 79-98.

2. G. W. Mackey, Unitary representations of group extensions. I, Acta Math. 99 (1958), 265-311.

MASSACHUSETtS INSTITUTE OF TEChNOLOGY AND

University of California, Los Angeles 